\title{
New carbon cone nanotip for use in a highly coherent cold field emission electron microscope
}

\author{
Florent Houdellier*, Aurélien Masseboeuf, Marc Monthioux, Martin J. Hÿtch \\ CEMES-CNRS, 29 rue Jeanne Marvig, BP 94347, TOULOUSE, Cedex 4, FRANCE
}

\begin{abstract}
A new cathode for cold-field emission gun using a pyrolytic carbon-cone supported onto a carbon nanotube as the electron emitting tip has been developped. This tip was mounted in a TEM using a FIB based method, and the brightness measured under real operating conditions is 5 times better than obtained with a standard tungsten tip. Its use overcomes the many technical difficulties which have dogged the use of carbon nanotube-based tips as proposed replacements for tungsten tips. The resulting properties of the final CFEG exhibit a very good energy spread of $0.32 \mathrm{eV}$, a reduced brightness of $1.6 \times 10^{9} \mathrm{~A} \cdot \mathrm{m}^{-2} \cdot \mathrm{sr}^{-1} \cdot \mathrm{V}^{-1}$ and a very good long-term stability with a current damping less than $16 \%$ per hour.
\end{abstract}

\section{Introduction}

The recent spectacular improvements in instrumentation for transmission electron microscopy, notably aberration correction [1-3], have highlighted the relative lack in progress of an essential part of the microscope column, i.e. the electron gun itself. The highest brightness electron guns are based on cold-field emission from tungsten tips, a technology strongly improved from the electron optics and vacuum point of view but still using the same cathode materials [4]. Until now, most of the latest generation electron microscopes are equipped with so-called Schottky

\footnotetext{
* Corresponding author: Fax : +33 562257999.

E-mail address: florent.houdellier@cemes.fr (F. Houdellier)
} 
field emission guns based on thermally field-assisted emission which are generally brightnesslimited by coulomb interactions $[5,6]$. The development of a cold field-emission electron source with higher brightness and coherence would benefit electron microscopy techniques across the board from high-resolution imaging [7] and atomic-resolution electron-energy-loss spectroscopy (EELS) [8], to electron holography $[9,10]$.

Indeed, electron sources for electron microscopy can be divided into two main families known as thermionic and field emission [5]. A thermionic gun uses a current-induced thermal excitation to allow electrons to pass over the energy barrier between the metal tip (usually a $\mathrm{LaB}_{6}$ single crystal) and the vacuum. The high emission currents are due to the fact that electrons are emitted from area from the tip. This makes these guns suitable for a wide range of conventional electron microscopy techniques. However, spatial and temporal coherence is poor due to the large virtual source size and the wide energy spread of the emitted electrons. This prevents their use as a source for electron holography or high-resolution electron energy loss spectroscopy. Field-emission guns (FEG) overcome these limitations by significantly decreasing the emission area (to about $50 \mathrm{~nm}$ diameter) and increasing the spatial coherence of the beam. Electrons are extracted by a strong electric field which enables them to tunnel through the energy barrier. However despite very good coherence (both spatial and temporal), the current decreases continuously during emission due to the adsorption of atoms (from the residual species in the vacuum or from degasing of the anode) onto the emitter surface. The surface can be cleaned by applying a burst of heat to the tip (called "flashing"), which can perturb experiments. Hence, FEGs are foremost restricted to scanning TEM instruments dedicated to electron spectroscopy and commonly named CFEG (for Cold Field Emission Gun). Schottky emitters are a hybrid combination of both above-described families more like a field-assisted thermionic emission than a genuine field emission [6]. A reasonable compromise is thus obtained between spatial coherence and beam current leading to a brightness which is higher than thermionic emitters but lower than cold-field emission. This compromise solution avoids the main disadvantages of a 
cold-field emitter and most electron microscopes which need a high brightness electron beam are equipped with such a gun. However, CFEGs remain superior for advanced spectroscopy as well as for specific TEM modes such as electron holography. Hence, pushed by the strong customer's demand to overcome the difficulties inherent to CFEGs, new vacuum and gun technologies are currently proposed by various TEM companies (namely Nion, Jeol and Hitachi) which allow flashing the tip not more than once a day or enable operators to clean the tip during the experiments without having to change the operating conditions, i.e. the flashing may be made under high voltage environment. However, in all nowadays new CFEG technologies on the market, the cathode material, made of tungsten, remains unchanged. The most promising candidates for replacing the tungsten tips used in cold-field emission are carbon nanotubes (CNTs) [11], which were demonstrated some years ago to be able of brightness one order of magnitude higher than tungsten tip as measured in an optical bench [12]. However, since then, such sources have not been successfully integrated in a high-voltage transmission electron microscope because of technical difficulties. Only low-accelerating voltage scanning electron microscopes (SEM) with modified Schottky guns were investigated [13,14]. The nanotube needs to be correctly mounted onto the filament which includes the laborious task of selecting a CNT which is suitably straight, aligning the selected CNT in the right direction for emission, and fixing the CNT so as to insure a good electrical contact. Reproducibility is poor due to the difficulty of the mounting operation [15] and the variation in field-emission characteristics and conductivity of the CNTs [16]. Growing a single, well-aligned CNT at the right place is tricky as well. A review of various procedures for such delicate mounting can be found elsewhere [17]. Furthermore, nanotubes are long and thin making them prone to vibrate [18], a feature which is able to provoke their breakage during emission [19] and a loss of spatial coherence for the electron beam extracted. The nanotube low diameter also limits the electron current that can be driven through the whole length of the tube, due to Joule heating [20]. Because of all these 
points, the efficiency of CNTs for cold-field emission in a high-voltage TEM has actually not been demonstrated so far.

Here we present the development of a new cathode for cold-field emission gun using a carbon nanotube-supported pyrolytic carbon cone [21-24], as a superior alternative to both standard tungsten tips and carbon nanotube tips. The relatively easy procedure to mount the carbon tip as the emitting tip is described, and the superior performances of the tip, as investigated both in a test-bench and in a TEM, are described a well.

\section{Experimental}

The carbon cones used in the study are prepared by a so-called "time-of-flight chemical vapour deposition" process which consists in depositing pyrolytic carbon onto previously grown carbon nanotubes used as substrates [21]. The overall morphology of the carbon objects used in the study is actually more complex than a mere carbon cone, as it can be precisely described as a carbon-nanotube-supported pyrolytic carbon deposit which includes a short microfiber segment with rough surface and two opposed cones with smooth surface which terminate the short fibre segment ends (Figure 1). However, the objects will be referred as "carbon cones" throughout the paper for practical use. Synthesis conditions and formation mechanisms were extensively described earlier as well as the various cone-bearing carbon morphologies which are able to be prepared by this process [22-24], including those used here. Briefly, the formation of the cone morphology is assumed to require the transient formation of specific hydrocarbon-rich droplets in the gas phase, whose average size with respect to that of the nanotube diameter should be such that they make possible the conditions for partial wetting [24]. 

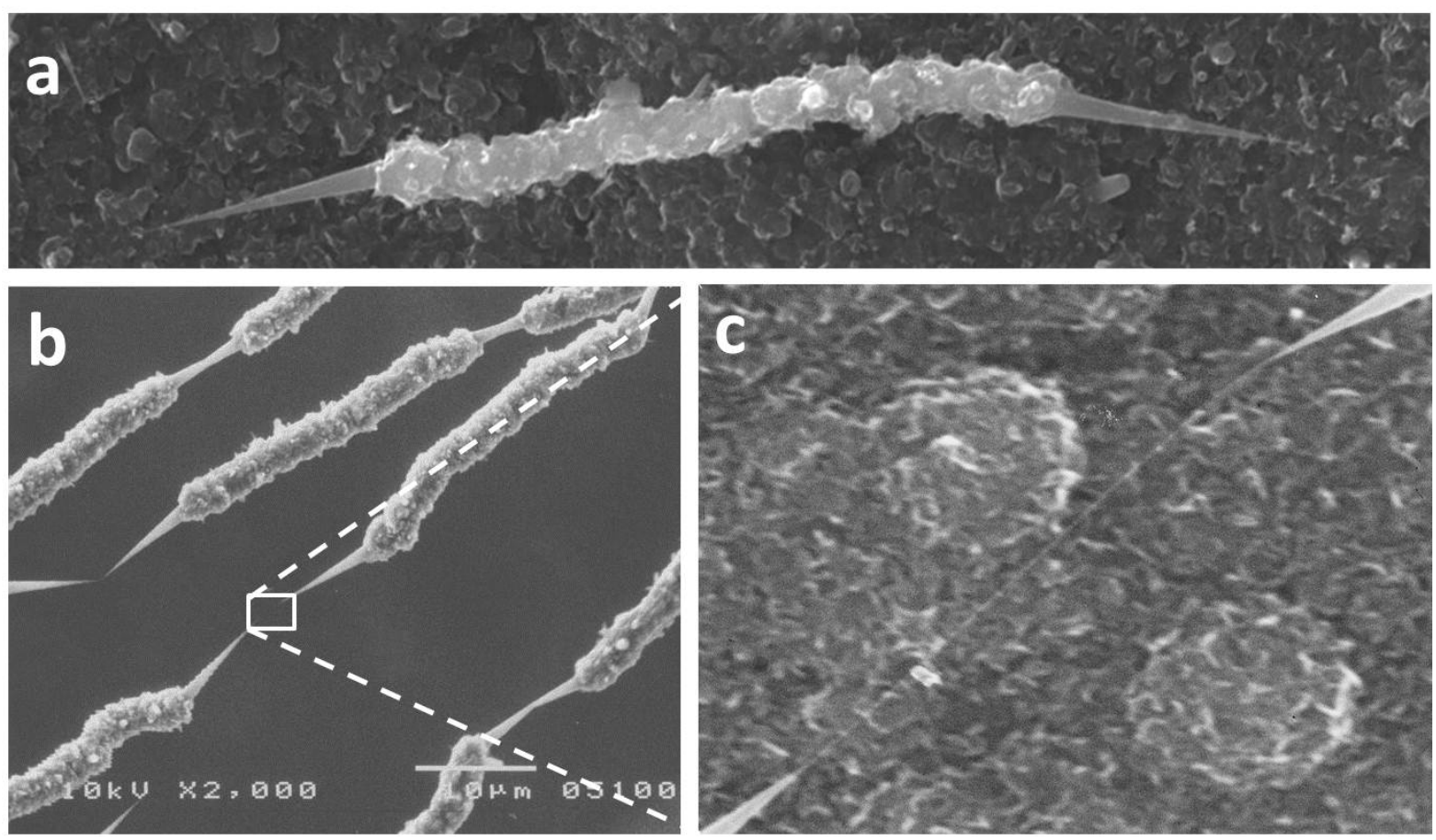

Fig. 1 - Scanning electron microscopy images. (a) Typical example of the carbon morphologies used in this work. The diameter of the short microfibre with rough surface between the two opposed cones is about $1 \mu \mathrm{m}$. (b) Example of similar morphologies also obtained, yet more thickened (the diameter of the short microfibers is about $4 \mu \mathrm{m}$ ). (c) High magnification of the area framed in (b). A non-thickened part of the supporting carbon nanotube (whose diameter is $\sim 5 \mathrm{~nm}$ ) shows up between two cone tips facing each other from two successive pyrolytic-carbon morphologies.

A tungsten tip is first prepared using a standard procedure, namely the micro-welding of a [310]oriented tungsten wire on a regular V-shape TEM tungsten filament. The tip of the tungsten wire is then electrochemically etched in a $\mathrm{KOH}$ solution. The resulting tungsten support can be illustrated by what is shown on right-hand in the inset of Figure 2D.

Afterwards, the various steps for mounting a carbon cone onto the home-made [310]oriented tungsten tip are carried-out in a dual Focused Ion Beam/FEG Scanning Electron Microscope (FIB/SEM) fitted with a nano-manipulator, as summarised in Fig. 2. 

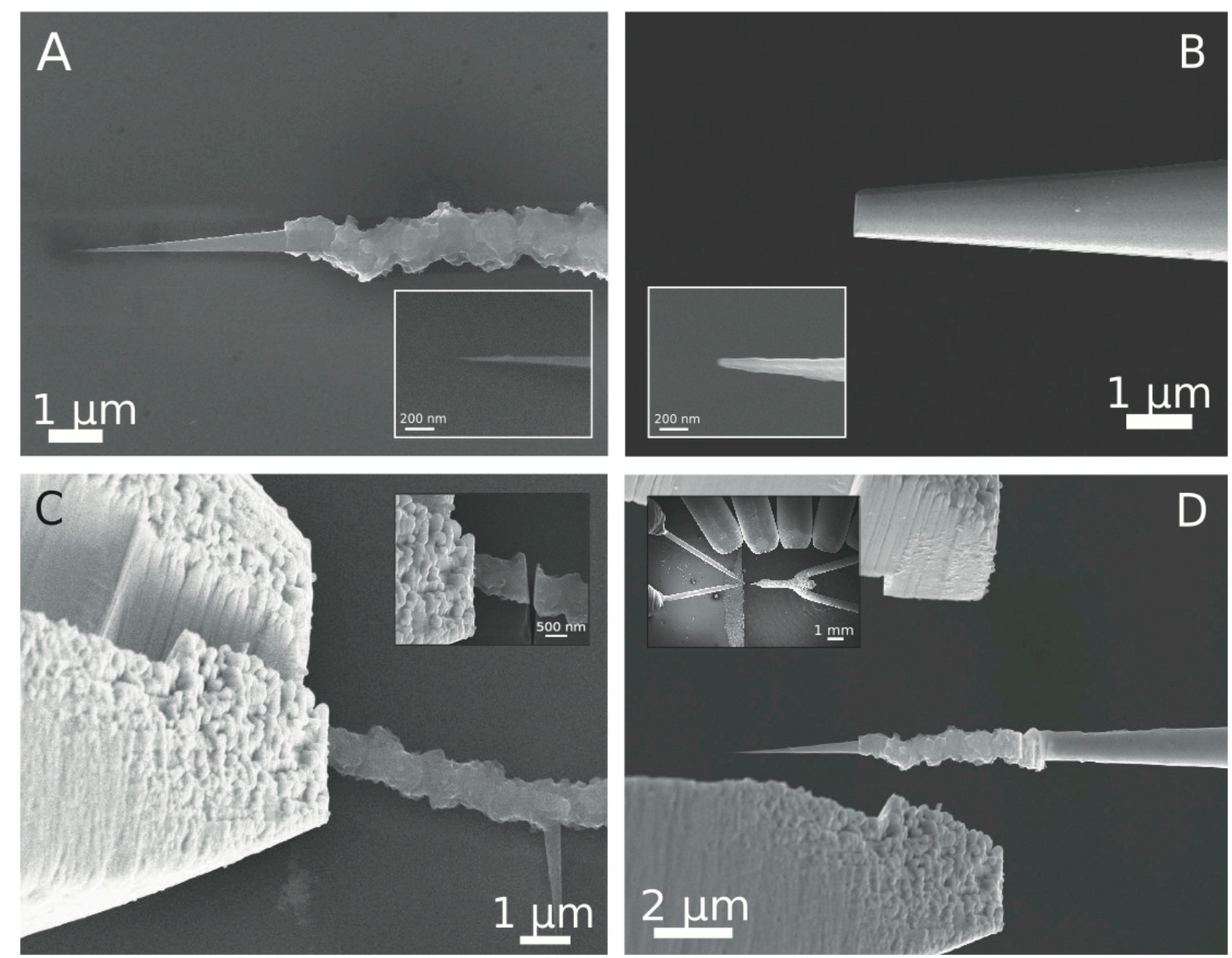

Fig 2 - Mounting procedure for the carbon cone tip. (A) View of part of the starting carbon object (similar to that in Figure 1a). The inset shows the apex radius of the carbon cone, below $10 \mathrm{~nm}$. (B) View of the tungsten tip after being truncated using the FIB. The inset shows the original apex of the tip. (C) The clamp of the carbon cone made with the tweezers at a $20^{\circ}$ tilt of the stage enables a protection of the tip during electron and ion imaging and ablation. The inset shows the next step when the carbon microfiber is cut using the FIB. (D) Resulting new tip after welding the carbon microfiber to the tungsten tip. The inset displays the whole set-up inside the dual beam (tweezers on the left, the new tungsten-supported carbon CFEG tip on the right, Gas injection nozzles on top)

A suitable carbon cone (Fig. 2A) is selected under SEM according to the proper alignment of the cone axis with respect to the microfiber portion axis. It is then lifted from the surface using micro-tweezers after cutting it from its base using a Ga ion beam (see Fig. 2C). The apex of the tungsten tip formerly prepared is also truncated by means of the Ga ion beam (Fig. 2B). The cut face of the carbon microfiber segment bearing the cone is then welded by $\mathrm{W}$ ion beam induced- 
deposition (from a $\mathrm{W}$ precursor, namely $\mathrm{W}(\mathrm{CO})_{6}$ ) to the truncated tungsten tip apex (Fig. 2D). An original CFEG shape is thus obtained displaying an apex radius below $5 \mathrm{~nm}$. A particular attention is paid to protect the carbon cone from parasitic deposition or ion irradiation of the cone by tilting the stage by 20 degrees. This enables a protection of the cone from the ion beam by the tweezers during the whole procedure (as can be seen on Fig. 2C). Indeed, TEM investigations of the carbon tip after mounting provided images similar to that in Fig. 3A taken on an as-grown cone, showing that the carbon cone structure was unaffected by any of the inFIB irradiation steps. The whole mounting procedure is robust, reproducible, and takes only two hours (including $30 \mathrm{~min}$ for preparing the supporting tungsten tip). It is worth noting that the same positioning, aligning, and fixing steps are required when attempting to use a pristine single carbon nanotube as emitting tip, but they are much facilitated by the specific multi-part morphology used here. Indeed, thanks to the short microfiber body, the object can easily be grabbed and welded, and thanks to the cone-microfiber self-alignment, the carbon cone can also be suitably positioned in spite of the nanometre size of the cone tip that will be the emitting part. 

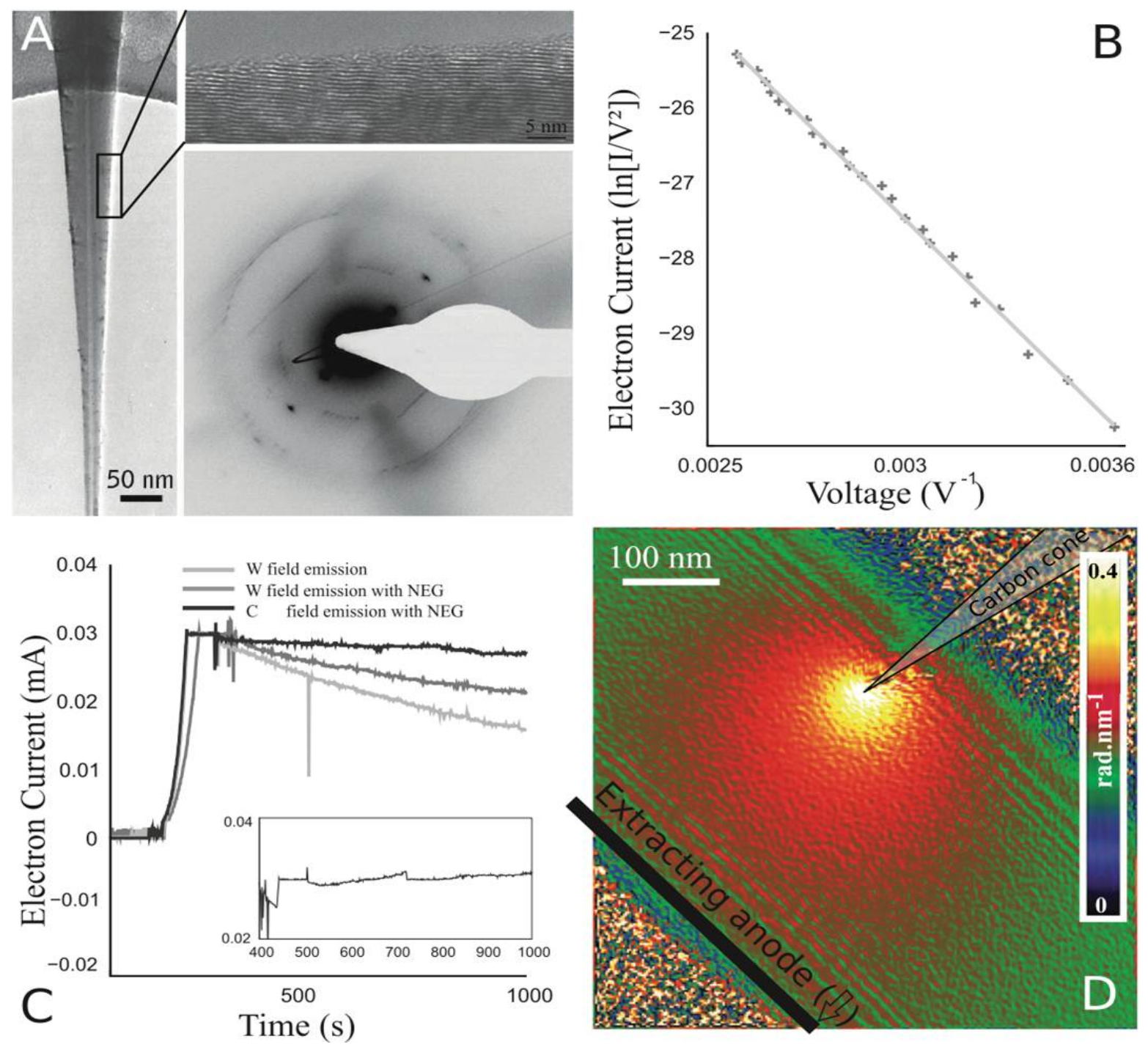

Fig. 3 - Characteristics of the carbon cone. (A) Medium (left) and high (top right) resolution TEM images of a carbon cone. The inner cavity of the supporting nanotube is seen. The carbon cone is made from graphenes with good nanotexture displayed concentrically as in MWCNTs, with the difference that the larger the graphene cylinder, the shorter its length. The bottom right image displays the associated diffraction pattern confirming that the cone is made of concentric graphene cylinders with perfect orientation with respect to the cone axis. See also [23]. (B) Fowler-Nordheim plot of the emission obtained inside the point projection chamber. (C) Emission stability for three configurations in the dedicated microscope: with a commercial tungsten tip; same tip after adding the NEG pumping system; with the carbon cone tip (and NEG pumping system). The inset displays emission for the carbon cone where small increases of current are observed. The inset time scale is in minutes (see text). (D) Holographic measurement during in-situ field-emission. The positions of both the carbon cone tip and of the extracting anode have been schematically reported on the image. The map displayed corresponds to the derivative of the hologram phase and is directly proportional to the electric field modulus around the tip. 
To fully characterize the emission properties of the new tip, two emission test benches were used. One is a home-made ultra-high vacuum (UHV) point projection microscope, in which the vacuum was optimized with the use of a special Non-Evaporated Getter (NEG) pumping system [25] in addition to the regular ion getter pump, enabling to reach vacuum beyond the detection limit of the gauge used, in the range of $10^{-10} \mathrm{~Pa}$. Indeed, the high pumping speed of the NEG pump can reduce the tip contamination due to anode degasing. The gun assembly was designed based on the ceramics and extracting anode of a former Philips EM400 cold-FEG module. A special degasing device for the extracting anode was designed using four halogen lamps placed around the gun assembly. Electrons are detected using a Faraday cup and a special transimpedance electronic circuit is used to convert the small current detected into a voltage signal directly captured by a National Instrument acquisition card. The total acceleration of the emitted electrons is then given by the extraction voltage. The signal is directly processed by a homemade Labview-based software.

The second bench used was a twenty-year old Hitachi HF2000 FE-TEM, in which the new tip was mounted to study the emission properties under high voltage environment. In order to achieve the correct vacuum condition, as for the UHV point projection microscope, the initial HF2000 vacuum circuit was modified by adding a NEG pump. The entire system was baked to achieve a suitable vacuum, better than $2 \cdot 10^{-9} \mathrm{~Pa}$. As shown in Figure 3C, such high vacuum drastically improves the emission properties when studying the slope of the current emitted by a standard tungsten tip. Furthermore, the vacuum below the gun valve was also improved to avoid degasing when opening the valve. However, the old electron optics technology used around the tip was left unchanged due to its difficult access. A Faraday cup was also installed in the beam stop position to measure the beam current, and a home-made $2 \mathrm{k} * 2 \mathrm{k}$ camera was mounted on a GIF200 (Gatan Image Filter) to capture the Fresnel fringe images, the electron holograms, and the zero-loss peak shape. The conditions to perform the Fresnel fringe patterns used to study the 
coherence properties of the beam were set with an emission current of $8 \mu \mathrm{A}$ inside the gun, an aperture size of $50 \mu \mathrm{m}$ and an exposure time of $1 \mathrm{~s}$. It is worth noting that the lens currents were left unchanged for both experiments (i.e., with the tungsten tip and the carbon-cone tip). To generate the electron holograms, a rotatable Moellensted biprism was positioned in the selected aperture plane of the objective lens. The zero-loss has been recorded using the GIF200 operated in the spectrometry mode with the smallest energy dispersion, a spectrometer entrance aperture of $0.6 \mathrm{~mm}$ and a condenser aperture of $50 \mu \mathrm{m}$. This spectrum has been captured with $0.3 \mathrm{~s}$ of exposure time using the diffraction mode of the TEM with a $0.2 \mathrm{~m}$ camera length and the incoming electron beam was set to $4 \mu \mathrm{A}$ inside the gun at $200 \mathrm{keV}$ accelerating voltage.

\section{Field emission properties of the carbon-cone tip}

As a consequence of our design, the current measured is that produced by the whole gun assembly. We were thus able to characterize the new tip in terms of field-emission properties and current stability, starting with a Fowler-Nordheim (FN) plot. The FN theory was formerly developed for planar emitters [26] but was extended to CNT emitters afterwards [27]. The FN plot (Fig. 3B) enables to recover the field-enhancement factor $\beta$ of the emitter which locally increases the macroscopic electric field mainly due to the shape of the emitter. It can be expressed without unit [28] or in relation with the anode-cathode distance [29,30]. Assuming a work function of $\Phi=5.0 \pm 0.1 \mathrm{eV}$ [29] a field enhancement factor of $\mathbf{1 5 0 0 0}$ is extracted from the FN plot. This value is much higher than for the standard tungsten tip and the extraction voltage is therefore much lower ( $400 \mathrm{~V}$ in this case). This is in the range of the giant fieldenhancement factor recently reported [31,32]. It could be explained considering the multistage form of our emitter [33]. This value was confirmed by in-TEM off-axis electron holography measurements (Fig. 3D) carried-out in a FEI TECNAI F20 TEM using a Nanofactory Instruments field-emission-dedicated double-tilt holder operated in Lorentz mode to map the 
electric field around the tip during emission using usual phase analysis [34]. A mean value of $2.10^{9} \mathrm{~V} \cdot \mathrm{m}^{-1}$ leading to a field-enhancement factor of 13000 , was obtained which confirms the Fowler-Nordheim results. Fig.3D also shows that the electrons are emitted from the very tip of the carbon-cone emitter.

\section{Improving the spatial coherence of a transmission electron microscope}

Electrons were extracted from the carbon tip to reach an intensity of $30 \mu \mathrm{A}$ and accelerated at $200 \mathrm{keV}$. As shown in the UHV point projection microscope, with the carbon nanocone tip, the strong field-enhancement factor lowers the extraction voltage needed to achieve the same emitted current as with a tungsten tip (1500 V in our case). Therefore, the ratio $\mathrm{R}$ between the extracting voltage and the focusing gun lens voltage was carefully adjusted for the carbon-cone tip to keep the maximum current at the end of the accelerating stage. In both cases (tungsten and carbon-cone) a distance of $7 \mathrm{~mm}$ was set from the tip to the extracting anode. Contrarily to a regular (tungsten-based) CFEG the current was extremely stable as shown in Fig. 3C. Moreover, it was found to slightly increase over several hours of emission (inset of Fig. 3C), and the extraction voltage needed to be lowered after one day of use. This side-effect was prevented by flashing the tip once before starting emitting in order to clean it from any adsorbed species, which is still by far much more convenient than flashing the tungsten tip every hour as with standard tungsten cathodes. However, even with the NEG pump, the high frequency emission noise was found in the same range as for the standard tungsten-tip, i.e. slightly higher than $1 \%$. This is believed to be a consequence of the old overall gun technology (extracting anode materials, tip relatively far from the pumping system linked by small holes in the extracting and focusing anodes, ...) which was the same for both the tungsten-tip and carbon-cone tip experiments. The reduced brightness of the carbon gun was measured with a Faraday cup which is the most accepted method [35]. A steady value of $\mathbf{1 . 6} \times \mathbf{1 0} \mathbf{A}^{\mathbf{A}} \cdot \mathbf{m}^{-\mathbf{2}} \cdot \mathbf{s r}^{-\mathbf{1}} \cdot \mathbf{V}^{\mathbf{- 1}}$ was found. 
The conditions used to determine the brightness were carefully chosen to minimize the diffraction effects and condenser lens aberrations as detailed in [35]. Thus, the current was measured equal to $2.5 \mathrm{nA}$ for a $0.3 \mathrm{~nm}$ probe size obtained in convergent beam mode with an opening angle of 6 mrad. Using the same method, a reduced brightness of $5 \times 10^{8} \mathrm{~A} \cdot \mathrm{m}^{-2} \cdot \mathrm{sr}^{-1} \cdot \mathrm{V}^{-1}$ only was obtained using the same gun assembly and operated with the standard tungsten tip. This clearly confirms the highly beneficial contribution of the carbon nanocone onto the reduced brightness of the overall gun assembly. This has also to be compared with values such as $1 \times 10^{8}$ A. $\mathrm{m}^{-2} \cdot \mathrm{sr}^{-1} \cdot \mathrm{V}^{-1}$ given for the most recent Schottky sources [36]. It is furthermore in the same range as what was expected for nanotube emitters [29]. In order to compare the coherence properties of the new tip with that of a tungsten tip, Fresnel fringes and electron holograms were recorded in the same conditions with the two tips. The use of off-axis electron holography to unambiguously compare the spatial coherence of the two sources is not straightforward due to the multiple perturbations that can induce a contrast damping. The Fresnel fringes of a defocused condenser aperture were then also recorded to compare the coherence degree of each source. The result is displayed in Figure 4. As explained in the experimental section, the conditions remained strictly identical for the two experiments. 

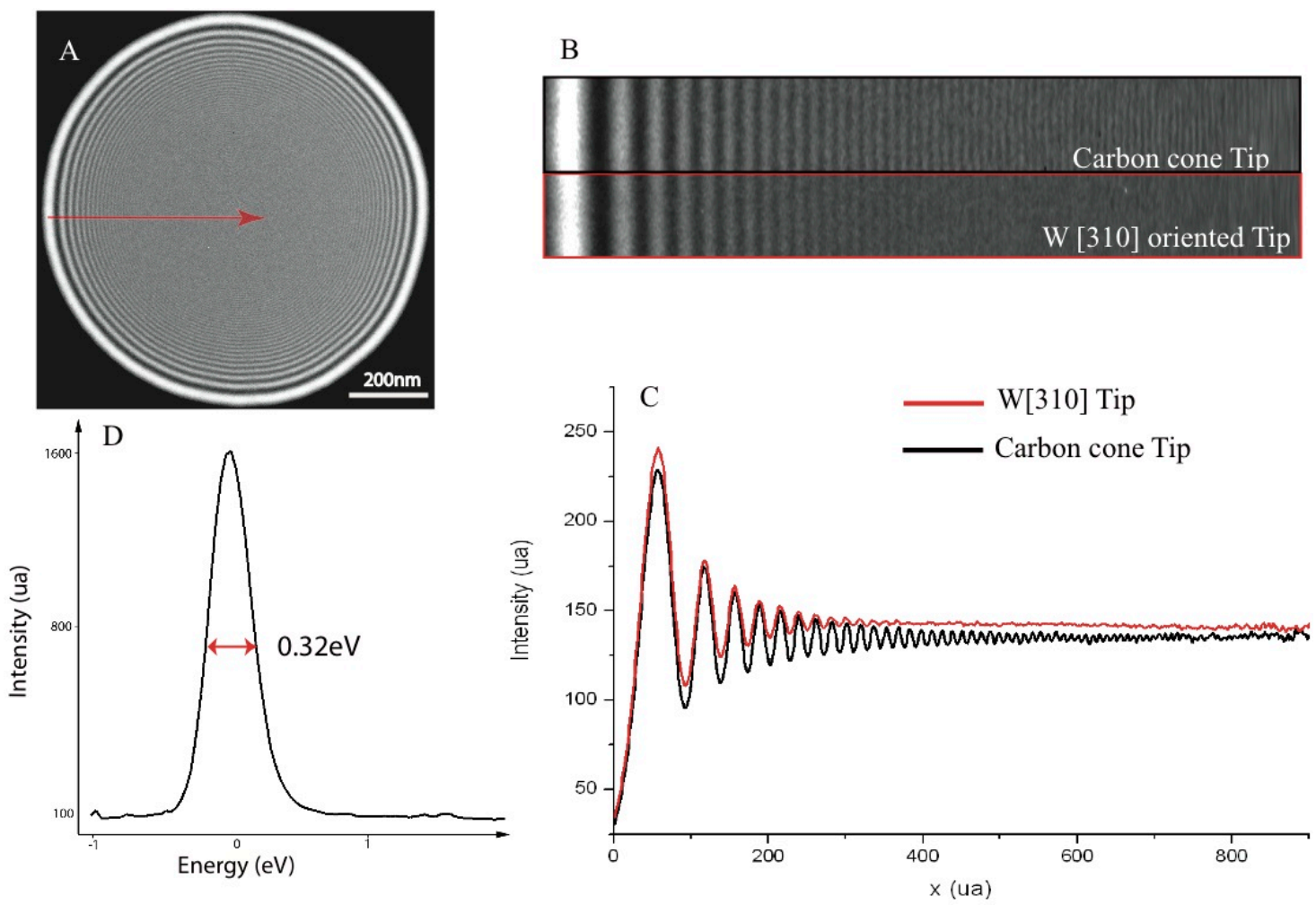

Fig. 4 - Spatial coherence and spectral properties obtained from the carbon cone mounted as the emitter in our CFEG TEM. (A) Fresnel fringes obtained from a defocused condenser aperture. (B) Comparison of the Fresnel fringe extension (angular sum) obtained with a commercial [310]-oriented tungsten tip and the new carbon cone source. (C) Fresnel fringe contrast averaged over the total angle of the condenser aperture obtained with the tungsten source (red) and the carbon cone source (black). (D) Energy spread obtained from the FWHM of the zero-loss peak of the electron energy loss spectrum obtained with the carbon nanocone electron source.

It clearly appears that the contrast of the Fresnel fringes is highly improved using the new carbon tip especially for distances far from the aperture edge. This unambiguous proof of the enhanced spatial coherence of the cold-field emission provided by the carbon cone opens many perspectives especially in the field of electron holography. Such a high brightness, high coherence CFEG source was used to perform standard off-axis electron holography experiments similar to that reported in [10]. We measured a contrast in electron holography of $54 \%$ with 100 $\mathrm{V}$ applied to the biprism wire compared to $40 \%$ obtained for this range of biprism voltage with 
Schottky sources [37]. These values are remarkable since the whole TEM equipment is over 20 years old and placed in a non-optimized (i.e., not free of parasitic fields) room.

The energy spread was also determined to be $\mathbf{0 . 3 2} \mathbf{e V}$ using the full width at half maximum (FWHM) of the zero-loss peak (Fig. 4D). This very good dispersion is in the same range as the best CFEG currently available [38]. Last but not least, our carbon tip has demonstrated to be fairly robust, with a life time now superior to 18 months (Figure 5), while its performances are still maintained.
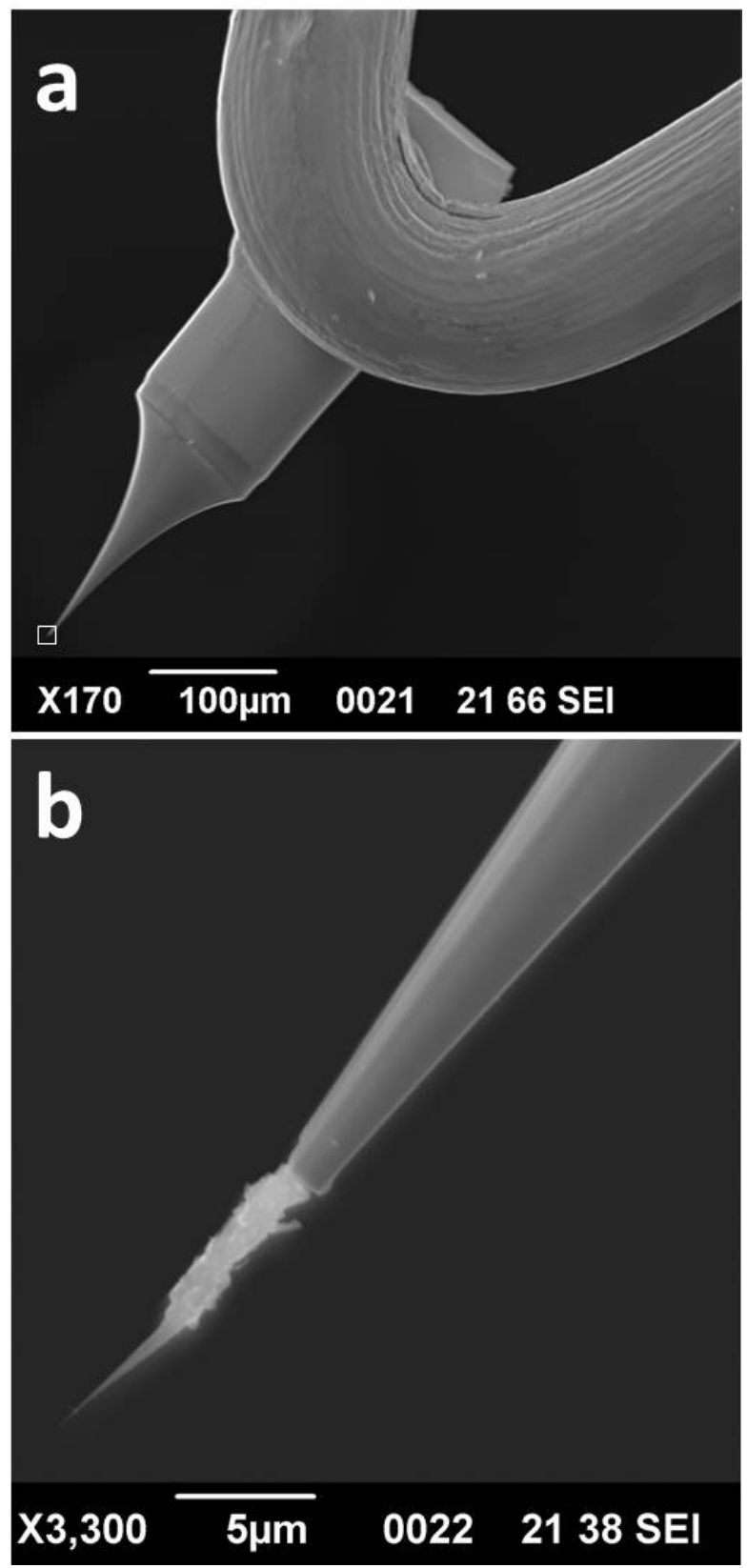
Fig. 5 - SEM images of the new tip after 18 months of use. (a) Overall view mostly showing the tungsten support, including the V-shaped tungsten wire with the etched tungsten tip welded to it. (b) Close view of the framed part of the image in (a) showing the carbon-cone tip still attached to the truncated tungsten tip support.

\section{Conclusions}

We have demonstrated that significant improvements of FEG-TEM performances (EELS, HRTEM, and holography) can be obtained by replacing the regular tungsten tip by a carbon cone tip prepared using specific CVD conditions. In particular, electron holography experiments could be performed with a much better signal over noise ratio in the reconstructed phase. As measured from the reduced brightness, field enhancement factor, current stability, energy spread, and spatial coherence, this new electron source technology makes a jump in CFEG performances with respect to tungsten-based emitters, in the same extent or better than carbon nanotubes were claimed to do. However, the specific morphology of the carbon-cone-bearing object used, which can be precisely described as "a carbon-nanotube-supported pyrolytic carbon deposit which includes a short microfiber segment with rough surface and two opposed cones with smooth surface which terminate the short fibre segment ends", overcomes the difficulties inherent to the use of pristine single carbon nanotubes, and make possible the actual technological transfer of such a new emitting carbon tip into TEMs. Hence, it brings out a real and robust application of carbon nanotube-based materials. Such a cathode associated with the latest generation of gun and TEM technologies should enable new developments with advanced electron interferometry and spectroscopy studies. Promising interferometry experiments are currently performed in our laboratory, exploiting the advantages brought by this new carbon-cone tip-equipped cold-field emission gun.

\section{Acknowledgements}


This work was supported by the European Union through the project ESTEEM (Enabling Science and Technology through European Electron Microscopy, IP3: 0260019) and the French

National Agency for Research (ANR) in the frame of its program in Nanosciences and Nanotechnologies (HDSTRAIN Project No. ANR-08-NANO-0-32). Pierre Stadelmann and Cecile Hebert from EPFL are warmly thanked for giving their HF2000 column to the CEMES. The authors would also like to thank Philippe Salles for help with the FIB preparation and Etienne Snoeck for critical rereading of the manuscript.

\section{References}

[1] Haider M, Uhlemann S, Schwan E, Rose H, Kabius B, Urban K. Electron microscopy image enhanced. Nature 1998;392:768-9.

[2] Krivanek OL, Dellby N, Lupini AR. Towards sub-angstrom electron beams. Ultramicroscopy 1999;78:1-11.

[3] Zach J. Chromatic correction: a revolution in electron microscopy, Philosophical Transactions of the Royal Society A 2009;367:3699-707.

[4] Crewe AV, Eggenberger DN, Wall J, Welter LM. Electron gun using a field emission source. Reviews of Scientific Instruments 1968;39:576-83.

[5] Hawkes PW, Kasper E. Principles of Electron Optics: Applied Geometrical Optics. Academic Press, 1989.

[6] Fransen MJ. Experimental evaluation of the extended schottky model for $\mathrm{ZrO} / \mathrm{W}$ electron emission, Journal of Vacuum Science \& Technology B: Microelectronics and Nanometer Structures 16 (4) (1998) 2063.

[7] Batson PE, Dellby N, Krivanek OL. Sub-angstrom resolution using aberration-corrected electron optics, Nature 2002;418:617-20. 
[8] Muller DA, Kourkoutis LF, Murfitt M, Song JH, Hwang HY, Silcox J, et al. Atomic-Scale chemical imaging of composition and bonding by aberration-corrected microscopy, Science 2008;319:1073-6.

[9] Lichte H, Geiger D, Linck M. Off-axis electron holography in an aberration-corrected transmission electron microscope. Philosophical Transactions of the Royal Society A 2009;367:3773-93.

[10] Hytch MJ, Houdellier F, Hue F, Snoeck E. Nanoscale holographic interferometry for strain measurements in electronic devices. Nature 2008;453:1086-9.

[11] Xu N, Huq S. Novel cold cathode materials and applications. Materials Science \& Engineering Reports 2005;48:47-189.

[12] de Jonge N, Lamy Y, Schoots K, Oosterkamp TH. High brightness electron beam from a multi-walled carbon nanotube. Nature 2002;420:393-5.

[13] Kruit P, Bezuijen M, Barth JE. Source brightness and useful beam current of carbon nanotubes and other very small emitters. Journal of Applied Physics 2006;99:024315.

[14] de Jonge N. Carbon nanotube electron sources for electron microscopes. Chapter 3 in: Advances in Imaging and Electron Physics, Elsevier, 2009, Vol 156, pp.203-33.

[15] de Jonge N, Lamy Y, Kaiser M. Controlled mounting of individual multiwalled carbon nanotubes on support tips. Nano Letters 2003;3:1621-4.

[16] Kim C-D, Jang H-S, Lee S-Y, Lee H-R, Roh Y-S, Rhee. I-S, et al. In situ characterization of the field-emission behavior of individual carbon nanotubes. Nanotechnology 2006;17:51804.

[17] Ribaya BP, Leung J, Brown P, Rahman M, Nguyen CV. A study on the mechanical and electrical reliability of individual carbon nanotube field emission cathodes. Nanotechnology 2008;19:185201.

[18] Saito Y, Seko K, Kinoshita J-I. Dynamic behavior of carbon nanotube field emitters observed by in situ transmission electron microscopy. Diamond and Related Materials 
$2005 ; 14: 1843-7$.

[19] Bonard J, Klinke C, Dean K, Coll B. Degradation and failure of carbon nanotube field emitters, Physical Review B 2003;67:115406.

[20] Vincent P, Purcell ST, Journet C, Binh VT. Modelization of resistive heating of carbon nanotubes during field emission. Physical Review B 2002;66:075406.

[21] Jacobsen RL, Monthioux M. Carbon beads with protruding cones. Nature 1997;385:211-2.

[22] Allouche H, Monthioux M, Jacobsen RL. Chemical vapor deposition of pyrolytic carbon on carbon nanotubes: Part I. Synthesis and morphology. Carbon 2003;41:2897-912.

[23] Allouche H, Monthioux M. Chemical vapor deposition of pyrolytic carbon on carbon nanotubes. Part II. Texture and structure. Carbon 2005;43:1265-78.

[24] Monthioux M., Allouche H. Jacobsen R. Chemical vapor deposition of pyrolytic carbon on carbon nanotubes. Part III: Formation mechanisms. Carbon 2006;44:3183-94.

[25] Audi M, Dolcino L, Doni F, Ferrario B. A new ultrahigh vacuum combination pump. Journal of Vacuum Science \& Technology A: Vacuum, Surfaces, and Films 1987;5:258790.

[26] Fowler R. H., Nordheim L. Electron emission in intense electric fields. Proceedings of the Royal Society of London Series A 1928;119:173-81.

[27] Liang SD, Chen L. Generalized Fowler-Nordheim theory of field emission of carbon nanotubes. Physical Review Letters 2008;101:027602.

[28] Bonard J, Croci M, Arfaoui I, Noury O, Sarangi D, Châtelain A. Can we reliably estimate the emission field and field enhancement factor of carbon nanotube film field emitters. Diamond and Related Materials 2002;11:763-8.

[29] de Jonge N, Allioux M, Oostveen JT, Teo KBK., Milne WI. Optical performance of carbonnanotube electron sources. Physical Review Letters 2005;94:186807.

[30] Smith RC, Forrest RD, Carey JD, Hsu WK, Silva SRP. Interpretation of enhancement factor in non-planar field emitters. Applied Physics Letters 2005;87:013111. 
[31] Chen G, Shin DH, Roth S, Lee CJ. Field emission characteristics of point emitters fabricated by a multiwalled carbon nanotube yarn. Nanotechnology 2009;20:315201.

[32] Kim HJ, Bae MJ, Kim YC, Cho ES, Sohn YC, Kim DY, et al. Growth of carbon nanotube field emitters on single strand carbon fiber: a linear electron source. Nanotechnology 2011;22:095602.

[33] Huang JY, Kempa K, Jo SH, Chen S, Ren ZF. Giant field enhancement at carbon nanotube tips induced by multistage effect. Applied Physics Letters 2005;87:053110.

[34] Cumings J, Zettl A, McCartney MR, Spence JCH. Electron holography of field-emitting carbon nanotubes. Physical Review Letters 2002;88:056804.

[35] Fransen MJ, Overwijk MHF, Kruit P. Brightness measurements of a $\mathrm{ZrO} / \mathrm{W}$ Schottky electron emitter in a transmission electron microscope. Applied Surface Science 1999;146:357-62.

[36] Van Veen AHV, Hagen CW, Bart JE, Kruit P. Reduced brightness of the ZrO/W Schottky electron emitter. Journal of Vacuum Science Technology B 2001;19:2038-44.

[37] Cooper D, Truche R, Rivallin P, Hartmann J, Laugier F, Bertin F, et al. Medium resolution off-axis electron holography with millivolt sensitivity. Applied Physics Letters 2007;91:143501.

[38] Krivanek OL, Dellby N, Keyse RJ, Murfitt MF, Own CS, Szilagyi ZS. Advances in aberration-corrected scanning transmission electron microscopy and electron energy-loss spectroscopy. Chapter 3 in: Aberration-Corrected Electron Microscopy. Advances in Imaging and Electron Physics, Vol. 153, Elsevier, 2008, pp.121-60. 